\title{
The Influence of Pregnancy on the Clinical Evolution and Prognosis of Pre-Existing Inflammatory and Autoimmune Skin Disorders and Their Management
}

\author{
Maria Rita Nasca Giorgia Giuffrida Giuseppe Micali \\ Dermatology Clinic, University of Catania, Catania, Italy
}

\section{Keywords}

Pregnancy · Outcome · Dermatoses · Inflammatory/

glandular/autoimmune skin disorders · Treatment

\begin{abstract}
Background: Although the possible occurrence of typical dermatoses during pregnancy is well recognized, little is known about the influence of pregnancy on the clinical evolution and prognosis of different pre-existing chronic dermatological disorders. Summary: In this study a comprehensive search of the available literature and reviews has been undertaken in order to collect and analyze articles reporting pre-existing chronic skin disorders in pregnant women and report current knowledge on their particular clinical and therapeutic aspects.

(c) 2020 S. Karger AG, Basel
\end{abstract}

\section{Introduction}

Pregnancy is a condition characterized by several biological changes in the endocrine, immunological and vascular systems essential for fetal attachment and survival. In pregnant women, such changes may result in the development of a wide range of both specific and ill-defined disorders affecting different organs and systems. Cutaneous manifestations observed during pregnancy are variable and can be classified into 3 groups: physiological cutaneous changes related to pregnancy, specific dermatoses of pregnancy, and flares or an exacerbation of pre-existing dermatoses [1-3]. Regarding this last group, although it is generally accepted that several skin conditions can typically occur or worsen in pregnant women, little is known about their clinical course and evolution; there is a paucity of data from the literature dealing with this subject. Furthermore, certain skin disorders may actually improve during pregnancy.

The aim of this study is to outline the influence of pregnancy on the clinical evolution and prognosis of different dermatoses based on the data from the available literature. Our findings may prove useful in the counseling of pregnant patients in a dermatologic setting.

\section{Materials and Methods}

Studies from the period between January 1,2000 and December 31, 2019 reporting chronic skin disorders in pregnant women were initially located on PubMed using the following key words: "pregnancy", "gestation", "skin disorders" and/or "disease". The titles and abstracts were collected and studies reporting specific dermatoses that typically occur during pregnancy were excluded. Those karger@karger.com

(C) 2020 S. Karger AG, Basel

www.karger.com/drm

Karger $\stackrel{2}{=}$
Giuseppe Micali

Dermatology Clinic, University of Catania

P.O. G. Rodolico, A.O.U. Policlinico "G. Rodolico - San Marco"

Via Santa Sofia 78, IT-95123 Catania (Italy)

cldermct@gmail.com 
reporting other pre-existing chronic skin disorders in pregnancy were specifically included. Subsequently, in order to further refine our retrospective evaluation and identify single pertinent disorders which could be relevant to the aim of our study, a new systematic electronic database PubMed search for each single disorder emerging from the full-text review of the articles obtained by the previous broader search was performed with no time limit restrictions. Pertinent literature cited in all the retrieved articles was also considered. The flowchart describing the screening process is shown in Figure 1. Selected skin disorders were finally grouped as follows: (1) inflammatory disorders; (2) inflammatory/glandular disorders; and (3) autoimmune disorders.

\section{Inflammatory Disorders}

\section{Psoriasis}

The current literature points towards an improving trend for the clinical course of psoriasis during pregnancy with a slight risk of flares following delivery [4-9].

Murase et al. [10] prospectively analyzed the clinical course of psoriasis during pregnancy and following delivery, and correlated progesterone and estrogens levels with disease changes in 47 psoriatic pregnant women. In accordance with previous research, they found that the proportion of women experiencing an improvement in their psoriatic disease was much greater than in those who experienced a worsening with $55 \%$ of patients reporting improvement, $21 \%$ no change, and $23 \%$ an exacerbation. Interestingly, the psoriatic body surface area showed on average an $83.8 \%$ decrease in pregnant women who experienced improvements, compared to a 5-20\% increase in those who reported an exacerbation in their condition. Following delivery, only $9 \%$ of patients reported an improvement; $26 \%$ reported no change, and $65 \%$ experienced a worsening of their condition. In these last cases, body surface area levels were close to those at the beginning of pregnancy, indicating a return of the condition to baseline, rather than an effective increase in its severity. It was also observed that high levels of estrogen corresponded to psoriasis improvement, whereas progesterone levels alone did not seem to correlate with psoriatic changes. Other studies reporting worsening within 6 weeks following delivery in about $40-90 \%$ of patients also supported these findings [11-13]. As regards fetal outcome, a negative impact is likely to result from concurrent comorbidities or drug exposure, rather than from a direct and potentially harmful underlying disease-related inflammation [11, 14-17].

Frequent remission during pregnancy of psoriasis, a primarily Th17-mediated disease, may be explained by the physiological maternal switch in the immune system to an adaptive Th2 response, characterized by a shift of the Th17/Treg ratio in favor of the Treg cells, that downregulate the effector immune response in order to allow a tolerance towards foreign fetal antigens [16]. Physiological hormonal factors (such as changes in progesterone, estrogen, and prolactin levels) probably play a key role in inducing pathogenetically relevant immunological and vascular changes both essential for fetal attachment and survival and affecting the course of disease [16].

For those patients experiencing disease exacerbation (about 10-20\%), evidence-based guidelines recommend the use of low- to mid-potency topical corticosteroids as first-line treatment for mild forms and narrow-band ultraviolet (NB-UVB) phototherapy as a second-line option. In severe psoriasis, cyclosporine and biologics may be considered as third-line options, carefully weighing the benefits and risks. Among biologics, certolizumab (approved only for psoriatic arthritis) has the most negligible rate of placental transfer. In newborns to mothers treated throughout pregnancy with biologic agents, live vaccination must be avoided for 6 months following delivery $[16,17]$.

A special mention should be reserved for impetigo herpetiformis, which is now regarded as a rare form of generalized acute pustular psoriasis triggered by pregnancy [18]. Its onset typically occurs in the last trimester of pregnancy with rapid resolution following delivery. It requires strict monitoring because its clinical course may lead to maternal or fetal mortality. It tends to recur with earlier onset and increased severity in subsequent pregnancies [18].

\section{Atopic Dermatitis}

Atopic dermatitis (AD) is considered the most common skin disorder occurring during pregnancy, accounting for $50 \%$ of dermatology referrals [19]. However, there is little knowledge on how pregnancy may affect the natural course of $\mathrm{AD}$, partly because the accuracy of differential diagnosis of eczematous eruptions in pregnancy itself may be challenging [20].

In previous studies, about $25 \%$ of women with $\mathrm{AD}$ have been shown to improve during pregnancy, whereas more than $50 \%$ had a worsening of their symptoms. A pre-existing eczema may worsen at any time during pregnancy, but it seems to occur more frequently in the second or third trimester $[21,22]$. In a study of 97 women, $14(61 \%)$ out of 23 patients who completed pregnancy reported a deterioration of $\mathrm{AD}$ symptoms during pregnancy, while 8 (35\%) remained unchanged, and 1 (4\%) noticed an improvement. In the 14 patients who experi- 


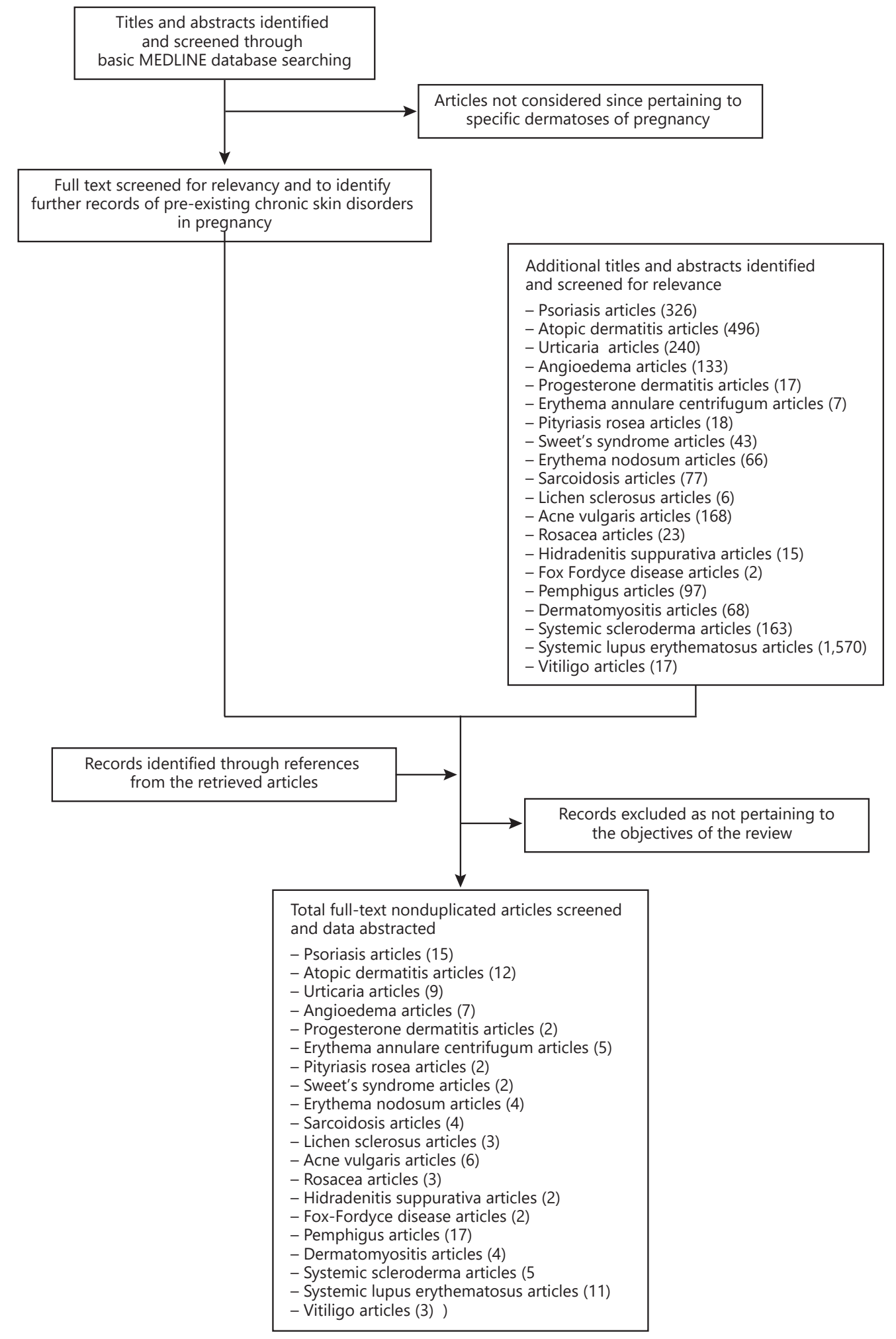

Fig. 1. Flowchart of the study selection process. 
enced worsening, $\mathrm{AD}$ severity was rated as mild in 2 (14\%), moderate in $8(57 \%)$, and severe in $4(29 \%)$. In most patients, exacerbations occurred during the second or third trimester (71\%) [22]. New-onset eczema may also develop in pregnancy, and it has been estimated that about $60-80 \%$ of patients develop symptoms for the first time during pregnancy, more often in the first and second trimester [23]. This last finding has raised the question whether pregnant women with new-onset disease may have true $\mathrm{AD}$ or transient eczematiform eruptions of unclear nosological classification that do not fully satisfy atopy criteria, resolve following delivery, and recur only in pregnancy. These include papular and prurigo-like rashes falling in the spectrum of the so-called atopic eruption of pregnancy (formerly known as prurigo gestationis, papular dermatitis of pregnancy, prurigo of pregnancy, and pruritic folliculitis of pregnancy) [19, 23-25].

The course of $\mathrm{AD}$ in pregnancy may be affected by hormonal factors, as suggested by a disease predominance in adult females, which is not observed in the prepubertal age [26], and by the reported perimenstrual exacerbation of AD in at least one third of women [27]. The shift towards a predominant Th2 response during pregnancy, which is known to be influenced by the hormonal background, may well explain worsening of the disease [28].

As regards the risk of fetal and maternal complications in $\mathrm{AD}$ pregnant women, no evidence of increased rates of infertility, prematurity, low birth weight, miscarriage, stillbirth, or birth defects has been found [29]. A recent study also excluded any association with maternal gestational diabetes and hypertension, whereas a nonstatistically significant increased risk of premature membrane rupture and of staphylococcal neonatal septicemia (which may be explained by higher rates of maternal perineal staphylococcal colonization in women with $\mathrm{AD}$ ) was reported [30].

Emollients and topical corticosteroids are recommended first-line treatments in pregnant women with AD. NB-UVB phototherapy and systemic corticosteroids may also be used if needed to control more severe cases [30].

\section{Urticaria}

Urticaria, sometimes associated with deeper tissue swelling (angioedema), may develop during gestation. In addition, the activity and severity of a pre-existing chronic spontaneous urticaria may either increase or improve [29]. Pressure urticaria often worsens [17]. However, precise data regarding the incidence and prevalence of urti- caria as well as its predictable clinical course during pregnancy are unavailable. Fertility, fetal development, labor, and delivery do not, however, seem to be significantly influenced by the disease [31]. Women are affected twice as often as men, and certain observations indicate that disease activity may be associated or triggered by fluctuation of sex hormone levels, including menstrual cycle, pregnancy, menopause, and hormonal therapies. One case report of urticaria unequivocally linked to pregnancy, confirmed by an intradermal estrogen injection test, has been reported [32]. However, the pathogenetic role of hormones in urticaria still remains controversial [33].

Importantly, when urticaria develops during gestation, some specific dermatoses of pregnancy, which may resemble common urticaria in their early stages, such as polymorphic eruption of pregnancy and pemphigoid gestationis, must be ruled out. Moreover, other dermatologic disorders, including progesterone dermatitis (PD) and erythema annulare centrifugum (that are further discussed below), as well as fixed-drug reaction, erythema multiforme, and contact dermatitis, should also be considered [34]. As regards polymorphic eruption of pregnancy, formerly known as pruritic urticarial papules and plaques of pregnancy, is the most common gestational dermatosis and is characterized by pruritic urticarial papules and plaques, normal laboratory tests, and negative direct immunofluorescence. It is more frequent in women at the end (third trimester) of their first pregnancy, and rarely occurs in the immediate postnatal period, with no tendency to recur in subsequent pregnancies [35]. On the other hand, pemphigoid gestationis is a rare disease which belongs to the pemphigoid group of autoimmune bullous disorders. It is characterized by a pruritic papular and vesiculobullous eruption that typically involves the abdomen and extremities and its onset is generally in the second or third trimester, although it may also occur following delivery [36].

Treatment with first- and second-generation antihistamines, such as $\mathrm{H} 1$ receptor antagonists, is considered safe in any trimester. In 2014 a systematic review of the literature found no correlation between prenatal antihistamine exposure and birth defects, with only 2 out of 31 cohort studies and 7 out of 23 case-control studies finding such significant association. Overall, the available data supporting the safe use of antihistamines on pregnancy are favorable, especially for loratadine, which has been extensively studied. However, there is still a need to assess safety with regard to population and ethnicity, since most research has gathered data from Western countries and Caucasians. More studies and data from different countries are needed to compare their use in different popula- 
tions [37]. Even though no definite teratogenic effects are reportedly associated with the intake of antihistamines in pregnancy, it should be noted that they are still not licensed by the FDA as category A safety group [38]. Moreover, antihistamines close to delivery are best avoided since they can increase uterine contractions. In breastfeeding women, discontinuation to avoid newborn exposure or, if unavoidable, use of second-generation antihistamines, such as cetirizine or loratadine, are preferable because they are present in lower levels in breast milk [39].

\section{Hereditary Angioedema}

Hereditary angioedema (HAE) is a rare autosomaldominant disorder characterized by recurrent, localized, and self-limiting acute edema episodes that, unlike acquired allergic angioedema, are unresponsive to conventional therapy with antihistamines, corticosteroids, or adrenaline. Three subtypes, 2 related to $\mathrm{C} 1$ inhibitor (C1INH) reduction (type I C1-INH-HAE) or dysfunction (type II C1-INH-HAE) and one showing no C1-INH deficiency (type III nC1-INH-HAE), have been identified. Concerning C1-INH-HAE, especially type III nC1-INHHAE, a female predominance has been observed, probably due to an influence of hormonal factors, such as estrogen exposure. Accordingly, a high number of patients have been reported to experience increased rates of attack during pregnancy in the more extensive published studies [40-42], especially in type III nC1-INH-HAE, although attacks may worsen, improve, or exhibit no change at all during pregnancy [40]. In addition, its severity during each single pregnancy may be different in the same patient. It may sometimes occur for the first time during pregnancy, a finding which is also more frequently observed in type III nC1-INH-HAE [31].

Most reports suggest more severe clinical symptoms and increased frequency of C1-INH-HAE attacks especially early during the first trimester, and mostly in women with an early disease onset in childhood [41, 43, 44]. Nevertheless, increased attack rates in the second and third trimesters have also been mentioned [45]. The abdomen is the most common area of localization, possibly as a result of mechanical pressure resulting from fetal growth and movements [45]. However, attacks rarely occur at delivery, despite the supposed unavoidable mechanical trauma [45].

C1-INH-HAE is characterized by an activation of the contact system which results in an increase in bradykinin formation and subsequently an increase in endothelial permeability, with vascular leakage and angioedema. A rapid raise of estrogen unbalanced by a consensual in- crease of progesterone levels has been claimed to overactivate the pathway, precipitating the acute attacks, especially in type III nC1-INH-HAE [46]. Mutations in the SERPING1 gene are responsible for type I and II C1INH-HAE, whereas some patients with type III nC1INH-HAE show mutations in the F12 gene, which encodes coagulation protein factor XII. Therefore, genetic testing may be a useful adjunct in the differential diagnosis of these disorders, especially when blood complement (C4, antigenic C1-INH, and functional C1-INH) level measurement yields false-positive results due to an increase in plasma volume during pregnancy.

In pregnant women with $\mathrm{HAE}$, some currently used treatments, such as antifibrinolytics and androgens, are strictly forbidden. Plasma-derived C1-INH may be used in the emergency management of life-threatening acute attacks, although type III HAE seems to be somewhat less responsive [45]. Safety data on the use of other drugs in pregnancy, such as icatibant or recombinant C1-INH, are still limited. Routine short-term prophylactic treatment before uncomplicated natural delivery is not necessary and is recommended only if forceps or vacuum extraction are used as well as in the case of cesarean section [45].

\section{Progesterone Dermatitis}

$\mathrm{PD}$ is a rare and highly invalidating acquired disorder mostly reported in women of childbearing age in their third decade of life. It typically relapses or worsens during pregnancy and around menses. In a review published in 2016, it was found to occur in relation to pregnancy in 13 out of 89 patients (14.6\% of cases), with 7 cases reported intrapartum and 6 following delivery [47]. PD is a hypersensitivity reaction triggered by the physiological changes of endogenous progesterone levels which is believed to result from a prior sensitization to progesterone leading to a T-cell-mediated immunological response. Predominant skin involvement is possibly explained by the expression of the progesterone receptor on epidermal keratinocytes. Whether sensitization occurs from exposure to exogenous progesterone, cross-reactivity from corticosteroids of the same hormonal group, or some other mechanism is still debated. In any case, it is likely that only low progesterone levels are tolerated in those women who develop the disease during pregnancy, when hormonal levels are well known to rise considerably [47].

PD clinical presentation may be highly heterogeneous, featuring urticarial, vesiculobullous, maculopapular, and purpuric eruptions. Therefore, several other skin disorders, such as urticaria, eczema, erythema multiforme, and vasculitis, should be considered in its differential diagno- 
sis. The most common symptom is pruritus. In addition, mucosal involvement, angioedema, respiratory distress, diarrhea, and anaphylaxis have sometimes been reported.

The diagnosis is strictly related to clinical history and may be confirmed by intradermal skin test to progesterone. Treatment is based on $\mathrm{H} 1$ and $\mathrm{H} 2$ antihistamines combined with topical and systemic corticosteroids for symptomatic relief. The only curative treatment is to decrease progesterone level by hormonal therapies. Treatment in pregnancy is clearly limited. Spontaneous remission is reported $[47,48]$.

\section{Erythema Annulare Centrifugum}

Only a few cases have been reported in pregnancy between the 12th and the 33rd week of gestational age [49, 50]. Its development, which shares pathogenetic similarities with chronic spontaneous urticaria, has been related to changes in hormone levels, with a consequent influence on the immunological and inflammatory response, triggering hypersensitivity. Estrogen [49, 50], human chorionic gonadotropin hormone [51], oxytocin, and prolactin have all been considered possible causes [52]. The role of these last 2 hormones is corroborated by the observation of transitory disease worsening early during breastfeeding [52]. Treatment is usually unnecessary. Pregnancy is uneventful and followed by spontaneous regression following delivery with no relapses [53].

\section{Pityriasis Rosea}

Pityriasis rosea has been reported to occur more frequently in pregnant women than in the general population (18 vs. 6\%), probably as a result of the physiological switch to a predominant Th2 immune response [17]. In a study on 38 pregnant patients who developed pityriasis rosea, miscarriage within the 17 th week and premature delivery occurred in 13 and $24 \%$ of patients, respectively, with a $16 \%$ rate of neonatal hypotonia in addition [54]. Women with an early onset of pityriasis rosea before week 15 and mucosal involvement were found to be at the highest risk for poor pregnancy outcome, followed by those with extensive skin involvement ( $>50 \%$ body area) and constitutional symptoms [55]. HHV-6 DNA may be detected in plasma, peripheral blood mononuclear cells, skin, and placenta and embryonic tissues by nested PCR, whereas serology tests disclose specific anti-HHV-6 and -7 IgM antibodies in only a minority of infected pregnant women [55].

Pityriasis rosea does not require a treatment since it is self-limited. Nevertheless, all pregnant women should avoid, especially during the first 15 weeks, any contact with patients known to have pityriasis rosea $[54,55]$.

\section{Sweet's Syndrome}

About 2\% of cases of Sweet's syndrome are reported to be associated with pregnancy [56]. Elevated estrogen and progesterone levels have been suggested to play a role by promoting vascular, cellular, microbiological, and immunological changes, leading to an excessive neutrophilic activation against undetermined self-components [56].

Pregnancy-associated Sweet's syndrome generally develops during the second or third trimester and may recur in subsequent pregnancies. Although miscarriage has been reported in one case, prognosis is favorable as this disorder is not associated with a significant increase of infant morbidity or mortality [56]. Treatment with a short course of systemic corticosteroids is highly effective, but may be avoided in most cases, since symptoms usually resolve spontaneously following delivery [57].

\section{Erythema Nodosum}

The correlation between erythema nodosum (EN) and female sex hormones is well known, with a proposed role of estrogen/progesterone ratio or high progesterone levels. In a prospective study on 132 patients, EN in pregnancy and after oral contraceptive use was reported in $10 \%$ of cases [58]. In addition, there have been several reports of EN occurring during pregnancy and after highdose combination oral contraceptive intake, as well as in postmenopausal women undergoing hormone replacement therapy or breast cancer patients treated with aromatase inhibitors [59]. The link between progesterone and $\mathrm{EN}$ is further supported by the case of a patient who developed EN after 4 weeks of intramuscular progesterone injections for assisted reproductive therapy, that resolved itself 2 days after discontinuing treatment [60].

Pregnancy is thought to create an optimal background for EN to develop and may act by inducing a hypersensitivity reaction to either estrogens or progesterone or, alternatively, causing the production of immune complexes playing a pathogenetic role. However, the exact mechanisms are unknown [61].

Normally, most cases of EN start in the first or early second trimester and resolve spontaneously by the end of the second trimester. Pregnancy is usually uneventful with no complications related to $\mathrm{EN}$, and fetal outcome is not considered at risk. Conservative treatment with elastic stockings and bed rest may be sufficient in most cases for symptom control [61].

\section{Sarcoidosis}

Conflicting opinions regarding how pregnancy may affect the course of a pre-existing sarcoidosis do exist. 
Some publications suggest a beneficial effect of pregnancy on sarcoidosis activity, with a possible exacerbation following delivery [62]. This notion is partly denied by controversial and contradictory findings in other research, showing either an improvement or, more often, a deterioration in the clinical course of the disease $[63,64]$.

As regards the influence of sarcoidosis on pregnancy, there is no evidence that sarcoidosis may affect fertility, and women are likely to experience uneventful pregnancies, with no significantly increased risk of abortions, low birth weight, or birth defects [62]. However, a retrospective study analyzing data from 678 patients with sarcoidosis found an increased risk of adverse events such as preeclampsia, eclampsia, deep vein thrombosis, pulmonary embolism, and premature delivery, with a higher rate of cesarean deliveries and postpartum hemorrhage, suggesting that thromboprophylaxis may be valuable in such patients [65].

Treatment of sarcoidosis during pregnancy depends on disease site and extent. Skin lesions may usually be managed with topical corticosteroids, restricting shortterm systemic use to acute flares.

\section{Vulvar Lichen Sclerosus}

The effect of pregnancy on the course of this typically chronic and relapsing disease has been little evaluated, with controversial results and inconsistent findings. In some studies, both worsening and improvement occurred [66]. These authors pointed to a likely influence of hormonal factors on disease course, based on their finding of a strict correlation of lichen sclerosus onset with the use of anti-androgenic oral contraceptives [67]. More recently, other researchers have concluded that pregnancy does not seem to cause any significant changes, based on the observation of similar average weekly doses of topical corticosteroids used by the patients before, during, and after pregnancy [68].

As regards treatment, the use of high-potency corticosteroids is not contraindicated and should not be withheld in pregnancy, as benefits outweigh the risks [68]. It is notable that current thinking does not support the need for cesarian section in pregnant women with lichen sclerosus [68].

\section{Inflammatory/Glandular Disorders}

\section{Acne Vulgaris}

Several studies have addressed the issue of the clinical course of acne vulgaris in pregnancy $[17,69-71]$.

Pre-Existing Dermatoses and Pregnancy
Initial studies suggested that pregnant women had an increased likelihood of acne regression. Improvement during pregnancy was observed in the majority of patients in 2 retrospective surveys, with 110 (57.5\%) out 191 patients and $20(41 \%)$ out of 49 patients reporting clinical amelioration, respectively $[69,70]$.

More recent studies report worsening of pre-existing acne in $35.5-51.5 \%$ of patients, mostly in the third trimester [71]. Clinically, inflammatory lesions confined to the face, mostly located in the mandibular area, have been observed [71]. Some authors have reported an association with primigravida, and female gender and low birth weight for gestational age of the newborn. However, current knowledge indicates that, overall, acne behavior in pregnancy remains unpredictable, and that association with several maternal (age, parity, and previous oral contraceptives intake) and fetal (gender and birth weight) features remains unsubstantiated [17].

Treatment of acne during pregnancy must take into consideration the potential fetal toxicity of many of the available therapeutic options, narrowing the choice only to drugs considered to be safe. Recommended first-line treatments include topical medications such as antibiotics (erythromycin, clindamycin, dapsone), benzoyl peroxide, and azelaic acid [72]. Salicylic acid is classified as FDA category $\mathrm{C}$ and there are no studies of topical salicylic acid supporting its safe use in humans during pregnancy. Malformations in rat embryos have resulted from systemic salicylic acid and aspirin administration during pregnancy. In addition, exposure in the third trimester may result in premature closure of the fetal ductus arteriosus. However, the risk related to exposure during pregnancy is considered minimal if its use is restricted to products containing low concentrations $(<2 \%)$ and applied sparingly to local areas for a limited duration of time $[73,74]$. The use of oral antibiotics ( $\beta$-lactams, macrolides) may be considered as second-line treatment, and oral prednisolone only for fulminant nodulocystic acne [73].

\section{Rosacea}

It is a commonly held opinion that rosacea often worsens during pregnancy, and that in some rare cases it may appear for the first time. However, there are no sound data available regarding the evolution of pre-existing rosacea in pregnancy, and therefore its course should be considered unpredictable [75]. Nevertheless, there are a number of anecdotal reports concerning 20 or so cases of rosacea fulminans occurring in pregnant women, mostly in the first or the third trimester and/or 
following delivery, with only one case complicated by intrauterine death and one other requiring pregnancy interruption [76].

Hormonal factors may play an important triggering role in this rare and severe variant of rosacea, as it has been observed also in women taking oral contraceptive pills, but definitive evidence is lacking $[76,77]$.

Although rare, rosacea fulminans in pregnancy remains a severely distressing disorder that poses therapeutic challenges related to the need for early and aggressive treatment limited by the contraindications of most treatments in pregnant women [77]. Besides topical antibiotics (metronidazole use is considered safe only in the third trimester), systemic erythromycin and corticosteroids, with close maternal and fetus monitoring, remain the mainstay of treatment [76].

\section{Hidradenitis Suppurativa}

There are only a few studies based on small sample sizes that have addressed the influence of pregnancy on the symptoms and course of hidradenitis suppurativa (HS), with most of them reporting improvement during pregnancy (20-83\% of patients) and worsening at menses (44-63\% of patients) [78]. This trend has also been confirmed by a recent survey-based cross-sectional study on a large series of women with HS who reported the influence of menses, pregnancy, or both on their symptoms [78]. Nonetheless, a systematic review has shown that a substantial subset of women has no alteration of HS disease course perimenstrually or during pregnancy [78], with only $20 \%$ of women experiencing symptom improvement during pregnancy in some series [79].

It has been suggested that during pregnancy, the relative increase of progesterone levels may play a protective role in at least a subset of HS patients by promoting differentiation of immune-modulatory Th2 and Treg cells, while suppressing the release of proinflammatory Th1/ Th17 cytokines. Conversely, the sudden premenstrual drop of these hormones may explain HS worsening around menses [78]. The role of a physiological apocrine activity decrease related to hormonal changes has also been taken into account.

Current guidelines do not address the management of HS in pregnant and breastfeeding women. Those showing persistent disease and requiring ongoing care may be managed using safer treatment modalities (antiseptic washes, topical antibiotics, intralesional corticosteroids), reserving systemic treatments (clindamycin/rifampin, dapsone, metformin) or limited surgical/laser excision to selected cases only [79].

\section{Fox-Fordyce Disease}

This rare inflammatory disorder of apocrine sweat glands typically affects women in child-bearing age (1535 years) and usually shows premenstrual flares and improvement during pregnancy (especially in the third trimester). These findings have corroborated the hypothesis of a strong, though still unproven, pathogenetic link with hormonal factors affecting postpubertal apocrine gland activity. However, recent studies regarding the subject are unavailable $[80,81]$.

\section{Autoimmune Disorders}

\section{Pemphigus}

Disease flares have been reported in pregnant women, in which pemphigus may be exacerbated or appear for the first time during pregnancy.

Two studies on 66 and 47 cases of pemphigus reported pemphigus vulgaris (PV) as the most common clinical variant (71-94\% of cases), observed either during or before pregnancy. In the last group, disease worsening was experienced in $54-62 \%$ of patients, with only $9.5-17 \%$ of cases reporting improvement, whereas about $29 \%$ of patients showed no change [82, 83]. In patients with multiple pregnancies, the disease was less severe or did not recur in later pregnancies.

Postpartum flares occurred more frequently (31-56\%) in cases with new onset during pregnancy, compared to those with pemphigus diagnosed before pregnancy, who showed lower rates of disease exacerbations following delivery [82, 83]. Following these extensive retrospective evaluations, only a few additional case reports have subsequently been published in recent years [84-88].

In conclusion, pregnancy as a triggering factor for pemphigus seems to be uncommon, whereas a pre-existing pemphigus may often exacerbate, remain stable or, less likely, enter remission during pregnancy. The risk of disease flares seems to be lower in women with a disease remission of at least 6-month duration with no therapy or minimal therapy [89]. Disease worsening is most likely during the first and second trimester, and postpartum period, whereas relief more often ensues during the third trimester.

The significant changes of the T-cell immune profile physiologically occurring during pregnancy probably play a crucial role in the development and evolution of various autoimmune diseases, including the new onset or worsening of a pre-existing pemphigus [89-91]. There is evidence that the Th1/Th2 balance becomes inclined to- 
ward production of Th2 cytokines for the period of gestation and, as pemphigus is a Th2-dominant autoimmune disorder, pregnant women are very likely to exhibit an increased occurrence $[91,92]$. In addition, the number of mast cells increases during pregnancy, and this could be related to the development and/or the exacerbations of pemphigus during pregnancy, although the exact role of mast cells in pemphigus has yet to be clarified [93].

Pemphigus flare following delivery has been reported to be due probably to the rapid drop of corticosteroid blood levels, whereas raised endogenous levels in the third trimester may be protective [83].

Disease activity correlation with an increased risk of abnormal pregnancy outcome (stillbirth, prematurity, spontaneous abortion, craniofacial malformations), although controversial, seems to be likely [89]. In addition, aberrant maternal autoantibodies are transmitted to the offspring, with possible transient neonatal development of blisters upon birth that usually spontaneously resolve within 4 weeks. Newborn lesions are more likely to occur with PV, whereas they are less often observed with pemphigus foliaceus due to the different desmoglein distribution in adult and fetal skin [83]. Children born to mothers with pemphigus appearing for the first time during pregnancy show lower rates of neonatal pemphigus [83]. Interestingly, no correlation has been found between the occurrence of neonatal pemphigus and maternal autoantibody levels, suggesting that they are of little significance as predictable markers of newborn disease risk [94, 95]. Furthermore, the reported finding of neonatal PV passively transmitted from clinically asymptomatic mothers unequivocally indicates that there must be other still poorly defined individual factors, besides antibody levels, facilitating disease outbreak $[96,97]$.

Management of pemphigus during pregnancy can be challenging and should take into consideration the severity of disease activity and medication safety concerns. Treatment discontinuation may be detrimental in women with the disease both for the mother and fetus. When the disease is progressive, low-dose systemic corticosteroids remain the mainstay treatment. Nonfluorinated corticosteroids (prednisone and prednisolone) should be preferred due to their limited transplacental passage and administered under close monitoring to prevent and promptly detect maternal or fetal complications. They may also be used in breastfeeding women, who should wait $4 \mathrm{~h}$ after oral administration before feeding. Patients of childbearing age should be advised to avoid pregnancy when they have recently experienced a relapse or are using certain drugs, such as azathioprine

Pre-Existing Dermatoses and Pregnancy
(AZA), mycophenolate mofetil (MMF) or methotrexate (MTX), that are not recommended or contraindicated in pregnancy and breastfeeding. MMF and MTX should be discontinued at least 3 weeks and 3 months, respectively, before pregnancy. In addition, folate should be prescribed early and taken throughout pregnancy in previous MTX users to reduce the risk of neural tube defects. Rituximab is generally not recommended and should be discontinued 12 months before pregnancy due to concerns regarding fetal B-cell development. Intravenous immune globulins or plasmapheresis/plasma exchange are considered safe in pregnancy and are thus recommended as second-line treatments when systemic corticosteroids fail to control severe and progressive disease $[89,98]$.

\section{Dermatomyositis}

The relationship of dermatomyositis and polymyositis with pregnancy is only addressed in a few studies [99]. Most retrospective analyses deal with a limited number of patients and report unfavorable fetal outcome, with a $50 \%$ fetal loss, when the disease appeared or showed a flare during pregnancy [100-102].

Based on the available data, optimal outcome can be anticipated when the pregnancy is undertaken with the disease in remission. Pre-existing disease does not seem to negatively affect pregnancy outcome unless a disease flare occurs during pregnancy. In this case, there is a high risk of complications both for the mother (hypertension, preeclampsia, eclampsia) [99] and newborn (miscarriage, prematurity, stillbirth), especially when worsening or relapse occur during the first trimester [102]. In these cases, disease activity may be controlled by increasing the corticosteroid dose, keeping in mind that close monitoring is mandatory to handle such pregnancies at high risk of poor outcome.

\section{Systemic Scleroderma}

The rarity of the disease, especially at a young age, explains the low frequency of concurrent scleroderma and pregnancy. Although a worsening during pregnancy of some disease symptoms, such as gastrointestinal upset, cardiopulmonary, and renal function impairment, may be observed, pregnancy does not seem to negatively affect the course of the disease. Peripheral perfusion improves probably due to increased cardiac output and lessened peripheral vascular resistance, and Raynaud's phenomenon and digital ulcers have been found to transiently regress after the second trimester, or at least to remain stable until the end of pregnancy [103]. 
Overall, maternal and neonatal survival is favorable, despite previous studies having shown high rates of miscarriage, premature birth, intrauterine growth restriction, and widely ranging rates of neonatal deaths, especially in cases with full-blown, long-standing, and extensive disease [103-105].

The disease has also been associated with increased maternal rates of hypertension and preeclampsia (prompting antenatal hospitalization and a longer hospital stay), and cesarean delivery compared to the general obstetric population [106].

Delay of pregnancy in women with recent-onset disease and avoidance in those with rapidly progressive severe organ damage is recommended, as the management of visceral complications may be difficult due to fetal toxicity drug limitations. General care during delivery may also be particularly challenging both for the gynecologist and for the anesthesiologist [104]. Ongoing multidisciplinary treatment, aimed to prevent active disease and compatible with fetal health (hydroxychloroquine, low-dose steroids, intravenous immunoglobulins), and close follow-up throughout pregnancy are mandatory $[104,107]$.

\section{Systemic Lupus Erythematosus}

Since systemic lupus erythematosus (SLE) develops at a relatively young age, affecting females more frequently than males (F:M = 7-15:1) and with no influence on fertility rates, its occurrence in pregnancy is not uncommon [108]. The immunological shift towards enhanced humoral and Th2 response in pregnancy may expectedly raise SLE activity. However, past available data regarding rates of disease flare in pregnant versus nonpregnant women with SLE have been controversial due to bias, including use of different diagnostic criteria and a therapeutic approach over a long time span [109].

In most studies, an increased incidence (57-61\%) of disease flare during pregnancy has been reported in patients with active disease [110-112], whereas in a recent report, out of a series of 500 pregnancies observed in a 2 -year period, only 3 cases $(0.6 \%)$ of pre-existing SLE worsening were observed [113].

The risk of SLE exacerbation is highest in the third trimester and puerperium (probably due to progressive increase in serum estrogen levels), and appears to depend mainly on the disease activity 6-12 months before conception, as women with quiescent disease over this period have less risk of flare during pregnancy (7-33 vs. 61-67\%) $[109,114]$. Therefore, it is advisable that patients with SLE have adequate disease control for at least 6 months before pregnancy [115].
Active SLE (with renal involvement, thrombocytopenia, antiphospholipid antibody positivity, and drop in complement levels) negatively affects pregnancy outcomes and is related to a high risk of preeclampsia, preterm delivery, pregnancy loss (miscarriage, stillbirth), and intrauterine growth restriction, especially in patients showing a more severe and advanced visceral disease. In addition, transient neonatal lupus due to passive transfer of maternal autoantibodies (especially with positive anti Ro/La antibodies) may occur, and rarely causes permanent and potentially fatal cardiac damage (heart block), peaking at 16-26 weeks of gestation [108, 109, 114, 116]. New-onset pregnant SLE patients are generally considered to experience more adverse maternal outcomes than active patients with SLE history [117]. Unlike women with systemic disease, those with exclusive mucocutaneous involvement have outcomes similar to those of healthy controls [118].

Close monitoring by a multidisciplinary team is mandatory in pregnant SLE patients. Complete treatment discontinuation during pregnancy may be detrimental and promote SLE reactivation. On the other hand, disease flares during pregnancy raise safety concerns for fetal health. Continued prophylactic treatment with antimalarials and pulse therapy with low-dose corticosteroids for flare control offer reasonable risk-benefit ratio and represent the preferred approach $[109,114]$. Selected patients may require immunosuppressive treatments selected among those considered to have less fetal toxicity (azathioprine, cyclosporine, tacrolimus). Intravenous immunoglobulin or plasmapheresis have been considered, although there is no agreement on an appropriate dose or schedule. As regards biologics (rituximab, belimumab), available safety data are still limited $[108,109,114]$. Patients with SLE and severe visceral involvement (pulmonary hypertension, advanced heart and/or renal failure, stroke) should be offered counseling for birth control, especially if at high risk. Hormonal oral contraceptives appear to be effective but may pose an increased risk of thromboembolic accidents for SLE patients positive for anti-phospholipid antibodies [114].

\section{Vitiligo}

A retrospective survey on 24 women with vitiligo, aimed at ascertaining disease activity prior to, during, and after pregnancy, found stable disease or improvement during pregnancy in most patients $(62.5$ and $12.5 \%$ respectively), with only $21 \%$ of patients reporting worsening. Only 1 patient reported disease onset during pregnancy. Increased cortisol and IL10 levels during pregnan- 
Table 1. Skin disorders reported to be affected by pregnancy and summary of available information regarding their clinical course and influence on maternal/fetal outcome

\begin{tabular}{|c|c|c|}
\hline Dermatosis & Disease course & Pregnancy outcome \\
\hline $\begin{array}{l}\text { Inflammatory disorders } \\
\text { Psoriasis }\end{array}$ & Improvement & Uneventful \\
\hline Atopic dermatitis & Worsening likely & Uneventful \\
\hline Urticaria & Unpredictable & Generally uneventful \\
\hline Hereditary angioedema & $\begin{array}{l}\text { Increased rates of attack that may worsen (especially type III), } \\
\text { improve, or exhibit no change }\end{array}$ & Generally uneventful \\
\hline Progesterone dermatitis & Relapses or worsen & Uneventful \\
\hline Erythema annulare centrifugum & Pregnancy may be a rare trigger & Generally uneventful \\
\hline Pityriasis rosea & Onset more frequent in pregnancy & $\begin{array}{l}\text { High risk of poor outcome, especially in case of } \\
\text { early onset }\end{array}$ \\
\hline Sweet's syndrome & Pregnancy may be a rare trigger & Generally uneventful \\
\hline Erythema nodosum & Pregnancy may be a frequent trigger & Generally uneventful \\
\hline Sarcoidosis & Improvement likely; possible worsening in postpartum & Poor outcome possible, but generally uneventful \\
\hline Vulvar lichen sclerosus & Potential improvement, but not explored in detail & Generally uneventful \\
\hline $\begin{array}{l}\text { Inflammatory/glandular disorders } \\
\text { Acne vulgaris }\end{array}$ & Unpredictable & No evidence \\
\hline Rosacea & Unpredictable; rosacea fulminans anecdotally reported & $\begin{array}{l}\text { No evidence; poor outcome likely in case of } \\
\text { rosacea fulminans }\end{array}$ \\
\hline Hidradenitis suppurativa & $\begin{array}{l}\text { Improvement likely in some reports but controversial } \\
\text { findings }\end{array}$ & No evidence \\
\hline Fox-Fordyce disease & Improvement likely, but no evidence & No evidence \\
\hline $\begin{array}{l}\text { Autoimmune disorders } \\
\text { Dermatomyositis }\end{array}$ & $\begin{array}{l}\text { Worsening likely, especially if active disease or }<6 \text { months' } \\
\text { remission }\end{array}$ & Poor outcome likely, especially if active disease \\
\hline Systemic scleroderma & Dependent on disease activity; possible improvement & Poor outcome possible, but generally uneventful \\
\hline Systemic lupus erythematosus & $\begin{array}{l}\text { Worsening likely, especially if active disease or }<6 \text { months' } \\
\text { remission }\end{array}$ & $\begin{array}{l}\text { Poor outcome likely; possible transient neonatal } \\
\text { disease due to passive antibody transfer }\end{array}$ \\
\hline Vitiligo & Improvement likely & Potentially affected but not explored in detail \\
\hline
\end{tabular}

cy have been suggested as possible factors underlying disease improvement [119].

As regards pregnancy outcome in patients with the disease, a retrospective comparative study analyzing data from 79 patients with vitiligo found no significant increase in obstetric risk factors, labor characteristics and complications, or birth outcome [120]. In contrast, a more recent large Korean retrospective cohort study on 4,738 pregnancies with vitiligo resulted in an association of vitiligo with an increased rate of spontaneous abortion. Upon subgroup analysis, the same study also showed more extensive disease to be associated with a higher risk [121]. However, other covariates (mother's lifestyle and comorbidities), potentially affecting pregnancy outcome, were not explored in detail.

Treatment options for vitiligo in pregnancy include topical corticosteroids and phototherapy. Folic acid supplementation should be especially recommended in pregnant women undergoing phototherapy.

\section{Conclusion}

Pregnancy is characterized by a series of important hormonal, immunological, and vascular changes. These modifications affect not only the skin, but the entire body 
requiring an adaptation to a new condition. Therefore, it is not surprising that most women, besides various physiological skin changes, may also experience improvement or worsening of pre-existing skin disorders or the onset of new conditions. The influence that pregnancy may have on the course of single dermatoses has occasionally been investigated, but only a limited number of reports on the outcome of chronic skin disorders during pregnancy are available so far (Table 1), and overall systematic evidencebased studies on large series of patients are still lacking. Further investigations would also be required to discover whether some skin disorders that are now considered pregnancy-specific (atopic eruption of pregnancy, impetigo herpetiformis) are only transient conditions that can occur exclusively in pregnancy or whether they should be regarded as diseases exacerbated or triggered by the gestational immunological and hormonal changes in predisposed pregnant individuals. Prospective, large-scale studies are mandatory to clarify these controversial issues.

The pathogenetic mechanisms underlying a modified disease course during gestation are also little investigated. Pregnancy is characterized by a decrease of cell-mediated maternal immunity (Th1-mediated) and a predominant humoral immunity (Th2-mediated). An estrogenic-driven shift of the immune system to an adaptive Th2 response may explain the remission during pregnancy of psoriasis and other inflammatory disorders, as well as the onset/ flare of Th2-supported diseases such as AD and PV. The evolution of other dermatoses may also be explained by other mechanisms. HS, for example, may improve during pregnancy also due to a hormonal-driven decrease of apocrine gland activity, while acne vulgaris worsening is probably related to a raise in androgen levels.

Special care should be dedicated to managing and preventing negative outcome deliveries, especially for those disorders such as pityriasis rosea, dermatomyositis/polymyositis, and SLE, that have been found to cause potential fetal damage. In this regard, proper pregnancy planning and safe therapeutic management (avoiding treatments harmful for the newborn) are mandatory.

In conclusion, a close collaboration between gynecologists and dermatologists and, when appropriate, a multidisciplinary approach also involving other specialties, such as rheumatology consultations, are always strongly advisable.

\section{Key Messages}

The clinical evolution and prognosis of pre-existing inflammatory and autoimmune skin disorders may be affected by pregnancy. The physiologic hormonal, immunological, and vascular changes that occur during pregnancy are likely to play a role, causing improvement or worsening of pre-existing skin disorders or the onset of new conditions.

\section{Disclosure Statement}

The authors have no conflicts of interest (including financial involvement and nonfinancial relationships) to declare.

\section{Funding Sources}

The authors have no funding sources to declare relevant to their study that may have potentially influenced the writing of the manuscript.

\section{Author Contributions}

All Authors participated with substantial contributions in the conception and design of the work; acquisition, analysis, and interpretation of data; drafting and critical revision of the work; and final approval of the manuscript.

\section{References}

1 Muallem MM, Rubeiz NG. Physiological and biological skin changes in pregnancy. Clin Dermatol. 2006 Mar-Apr;24(2):80-3.

2 Vora RV, Gupta R, Mehta MJ, Chaudhari AH, Pilani AP, Patel N. Pregnancy and skin. J Family Med Prim Care. 2014 Oct-Dec;3(4): 318-24.

3 Danesh M, Pomeranz MK, McMeniman E, Murase JE. Dermatoses of pregnancy: Nomenclature, misnomers, and myths. Clin Dermatol. 2016 May-Jun;34(3):314-9.

4 Johnson MA, Armstrong AW. Clinical and histologic diagnostic guidelines for psoriasis: a critical review. Clin Rev Allergy Immunol. 2013 Apr;44(2):166-72.
5 Coimbra S, Figueiredo A, Castro E, RochaPereira P, Santos-Silva A. The roles of cells and cytokines in the pathogenesis of psoriasis. Int J Dermatol. 2012 Apr;51(4):389-95.

6 Islam MT, Paul HK, Zakaria SM, Islam MM, Shafiquzzaman M. Epidemiological determinants of psoriasis. Mymensingh Med J. 2011 Jan;20(1):9-15.

7 Mowad CM, Margolis DJ, Halpern AC, Suri B, Synnestvedt M, Guzzo CA. Hormonal influences on women with psoriasis. Cutis. 1998 May;61(5):257-60.

8 Danesh M, Murase JE. The immunologic effects of estrogen on psoriasis: A comprehensive review. Int J Womens Dermatol. 2015 May;1(2):104-7.
9 Shelly S, Boaz M, Orbach H. Prolactin and autoimmunity. Autoimmun Rev. 2012 May; 11(6-7):A465-70.

10 Murase JE, Chan KK, Garite TJ, Cooper DM, Weinstein GD. Hormonal effect on psoriasis in pregnancy and post partum. Arch Dermatol. 2005 May; 141(5):601-6.

11 Lima XT, Janakiraman V, Hughes MD, Kimball $\mathrm{AB}$. The impact of psoriasis on pregnancy outcomes. J Invest Dermatol. 2012 Jan;132(1): 85-91.

12 Boyd AS, Morris LF, Phillips CM, Menter MA. Psoriasis and pregnancy: hormone and immune system interaction. Int J Dermatol. 1996 Mar;35(3):169-72. 
13 Ceovic R, Mance M, Bukvic Mokos Z, Svetec M, Kostovic K, Stulhofer Buzina D. Psoriasis: female skin changes in various hormonal stages throughout life-puberty, pregnancy, and menopause. BioMed Res Int. 2013 Dec; 2013:571912.

14 Seeger JD, Lanza LL, West WA, Fernandez C, Rivero E. Pregnancy and pregnancy outcome among women with inflammatory skin diseases. Dermatology. 2007 Dec;214(1):32-9.

15 Ben-David G, Sheiner E, Hallak M, Levy A. Pregnancy outcome in women with psoriasis. J Reprod Med. 2008 Mar;53(3):183-7.

16 Porter ML, Lockwood SJ, Kimball AB. Update on biologic safety for patients with psoriasis during pregnancy. Int J Womens Dermatol. 2017 Feb;3(1):21-5.

17 Yang CS, Teeple M, Muglia J, Robinson-Bostom L. Inflammatory and glandular skin disease in pregnancy. Clin Dermatol. 2016 MayJun;34(3):335-43.

18 Namazi N, Dadkhahfar S. Impetigo herpetiformis: review of pathogenesis, complication, and treatment. Dermatol Res Pract. 2018 Apr; 2018:5801280.

19 Vaughan Jones S, Ambros-Rudolph C, Nelson-Piercy C. Skin disease in pregnancy. BMJ. 2014 Jun;348:g3489.

20 Resende C, Braga A, Vieira AP, Brito C. Atopic eruption of pregnancy: a recent but controversial classification. Aust J Dermatol. 2014 Jun;1(3):1011.

21 Kemmett D, Tidman MJ. The influence of the menstrual cycle and pregnancy on atopic dermatitis. Br J Dermatol. 1991 Jul;125(1):59-61.

22 Cho S, Kim HJ, Oh SH, Park CO, Jung JY, Lee $\mathrm{KH}$. The influence of pregnancy and menstruation on the deterioration of atopic dermatitis symptoms. Ann Dermatol. 2010 May; 22(2): $180-5$.

23 Ambros-Rudolph CM, Müllegger RR, Vaughan-Jones SA, Kerl H, Black MM. The specific dermatoses of pregnancy revisited and reclassified: results of a retrospective twocenter study on 505 pregnant patients. J Am Acad Dermatol. 2006 Mar;54(3):395-404.

24 Koutroulis I, Papoutsis J, Kroumpouzos G. Atopic dermatitis in pregnancy: current status and challenges. Obstet Gynecol Surv. 2011 Oct;66(10):654-63.

25 Roth MM, Cristodor P, Kroumpouzos G. Prurigo, pruritic folliculitis, and atopic eruption of pregnancy: facts and controversies. Clin Dermatol. 2016 May-Jun;34(3):392-400.

26 Chen W, Mempel M, Schober W, Behrendt H, Ring J. Gender difference, sex hormones, and immediate type hypersensitivity reactions. Allergy. 2008 Nov;63(11):1418-27.

27 Kiriyama K, Sugiura H, Uehara M. Premenstrual deterioration of skin symptoms in female patients with atopic dermatitis. Dermatology. 2003;206(2):110-2.

28 Mor G, Cardenas I. The immune system in pregnancy: a unique complexity. Am J Reprod Immunol. 2010 Jun;63(6):425-33.

29 Woidacki K, Zenclussen AC, Siebenhaar F. Mast cell-mediated and associated disorders in pregnancy: a risky game with an uncertain outcome? Front Immunol. 2014 May;5:231.

30 Hamann CR, Egeberg A, Wollenberg A, Gislason G, Skov L, Thyssen JP. Pregnancy complications, treatment characteristics and birth outcomes in women with atopic dermatitis in Denmark. J Eur Acad Dermatol Venereol. 2019 Mar;33(3):577-87.

31 Kadar L, Kivity S. Urticaria and angioedema in pregnancy. Curr Dermatol Rep. 2013 Dec; 2(4):236-42.

32 Kasperska-Zajac A, Brzoza Z, Rogala B. Sex hormones and urticaria. J Dermatol Sci. 2008 Nov;52(2):79-86.

33 Lee AY, Lee KH, Kim YG. Oestrogen urticaria associated with pregnancy. Br J Dermatol. 1999 Oct;141(4):774.

34 Schaefer P. Acute and chronic urticaria: evaluation and treatment. Am Fam Physician. 2017 Jun;95(11):717-24.

35 Chouk C, Litaiem N. Pruritic urticarial papules and plaques of pregnancy (PUPPP). StatPearls. Treasure Island (FL): StatPearls Publishing; 2020.

36 Kukkamalla RM, Bayless P. Pemphigoid gestationis. Clin Pract Cases Emerg Med. 2019 Jan;3(1):79-80.

37 Gilboa SM, Ailes EC, Rai RP, Anderson JA, Honein MA. Antihistamines and birth defects: a systematic review of the literature. Expert Opin Drug Saf. 2014 Dec;13(12):166798.

38 Kar S, Krishnan A, Preetha K, Mohankar A. A review of antihistamines used during pregnancy. J Pharmacol Pharmacother. 2012 Apr; $3(2): 105-8$

39 Lawlor F. Urticaria and angioedema in pregnancy and lactation. Immunol Allergy Clin North Am. 2014 Feb;34(1):149-56.

40 Bouillet L, Longhurst H, Boccon-Gibod I, Bork K, Bucher C, Bygum A, et al. Disease expression in women with hereditary angioedema. Am J Obstet Gynecol. 2008 Nov;199(5): 484.e1-4.

41 Czaller I, Visy B, Csuka D, Füst G, Tóth F, Farkas H. The natural history of hereditary angioedema and the impact of treatment with human C1-inhibitor concentrate during pregnancy: a long-term survey. Eur J Obstet Gynecol Reprod Biol. 2010 Sep;152(1):44-9.

42 Martinez-Saguer I, Rusicke E, AygörenPürsün E, Heller C, Klingebiel T, Kreuz W. Characterization of acute hereditary angioedema attacks during pregnancy and breastfeeding and their treatment with $\mathrm{C} 1$ inhibitor concentrate. Am J Obstet Gynecol. 2010 Aug; 203(2):131.e1-7.

43 Winnewisser J, Rossi M, Späth P, Bürgi H. Type I hereditary angio-oedema. Variability of clinical presentation and course within two large kindreds. J Intern Med. 1997 Jan;241(1): 39-46.

44 Chinniah N, Katelaris CH. Hereditary angioedema and pregnancy. Aust N Z J Obstet Gynaecol. 2009 Feb;49(1):2-5.

45 Caballero T, Canabal J, Rivero-Paparoni D, Cabañas R. Management of hereditary angio- edema in pregnant women: a review. Int J Womens Health. 2014 Sep;6:839-48.

46 Feray S, Fain O, Kayem G, Sabourdin N, Constant I, Rigouzzo A. Repeated attacks of type III hereditary angioedema with factor XII mutation during pregnancy. Int $\mathrm{J}$ Obstet Anesth. 2018 Nov;36:114-8.

47 Nguyen T, Razzaque Ahmed A. Autoimmune progesterone dermatitis: update and insights. Autoimmun Rev. 2016 Feb;15(2):191-7.

48 Detrixhe A, Nikkels AF, Dezfoulian B. Autoimmune progesterone dermatitis. Arch Gynecol Obstet. 2017 Nov;296(5):1013-4.

49 Choonhakarn C, Seramethakun P. Erythema annulare centrifugum associated with pregnancy. Acta Derm Venereol. 1998 May;78(3):237-8.

50 Rosina P, D’Onghia FS, Barba A. Erythema annulare centrifugum and pregnancy. Int $J$ Dermatol. 2002 Aug;41(8):516-7.

51 Dogan G. Pregnancy as a possible etiologic factor in erythema annulare centrifugum. Am J Clin Dermatol. 2009 Aug;10(1):33-5.

52 Fuentelsaz V, Corredera C, Ara M, Carapeto FJ. [Pregnancy-related erythema annulare centrifugum]. Actas Dermosifiliogr. 2011 Jan; 102(1):66-8.

53 Chiang CH, Lai FJ. Pregnancy-associated erythema annulare centrifugum. J Formos Med Assoc. 2015 Jul;114(7):670-1.

54 Drago F, Broccolo F, Zaccaria E, Malnati M, Cocuzza C, Lusso P, et al. Pregnancy outcome in patients with pityriasis rosea. J Am Acad Dermatol. 2008 May;58(5 Suppl 1):S78-83.

55 Drago F, Ciccarese G, Herzum A, Rebora A, Parodi A. Pityriasis rosea during pregnancy: major and minor alarming signs. Dermatology. 2018;234(1-2):31-6.

56 Corbeddu M, Pilloni L, Pau M, Pinna AL, Rongioletti F, Atzori L. Treatment of Sweet's syndrome in pregnancy. Dermatol Ther (Heidelb). 2018 Jul;31(4):e12619.

57 Satra K, Zalka A, Cohen PR, Grossman ME. Sweet's syndrome and pregnancy. J Am Acad Dermatol. 1994 Feb;30(2 Pt 2):297-300.

58 Psychos DN, Voulgari PV, Skopouli FN, Drosos AA, Moutsopoulos HM. Erythema nodosum: the underlying conditions. Clin Rheumatol. 2000;19(3):212-6.

59 Jhaveri K, Halperin P, Shin SJ, Vahdat L. Erythema nodosum secondary to aromatase inhibitor use in breast cancer patients: case reports and review of the literature. Breast Cancer Res Treat. 2007 Dec;106(3):315-8.

60 Jeon HC, Choi M, Paik SH, Na SJ, Lee JH, Cho $\mathrm{S}$. A case of assisted reproductive therapy-induced erythema nodosum. Ann Dermatol. 2011 Aug;23(3):362-4.

61 Acosta KA, Haver MC, Kelly B. Etiology and therapeutic management of erythema nodosum during pregnancy: an update. Am J Clin Dermatol. 2013 Jun;14(3):215-22.

62 Selroos O. Sarcoidosis and pregnancy: a review with results of a retrospective survey. J Intern Med. 1990 Apr;227(4):221-4.

63 Agha FP, Vade A, Amendola MA, Cooper RF. Effects of pregnancy on sarcoidosis. Surg Gynecol Obstet. 1982 Dec;155(6):817-22. 
64 Haynes de Regt R. Sarcoidosis and pregnancy. Obstet Gynecol. 1987 Sep;70(3 Pt 1):36972.

65 Hadid V, Patenaude V, Oddy L, Abenhaim HA. Sarcoidosis and pregnancy: obstetrical and neonatal outcomes in a population-based cohort of 7 million births. J Perinat Med. 2015 Mar;43(2):201-7.

66 Helm KF, Gibson LE, Muller SA. Lichen sclerosus et atrophicus in children and young adults. Pediatr Dermatol. 1991 Jun;8(2):97101.

67 Günthert AR, Faber M, Knappe G, Hellriegel $S$, Emons G. Early onset vulvar lichen sclerosus in premenopausal women and oral contraceptives. Eur J Obstet Gynecol Reprod Biol. 2008 Mar;137(1):56-60.

68 Nguyen Y, Bradford J, Fischer G. Lichen sclerosus in pregnancy: A review of 33 cases. Aust N Z J Obstet Gynaecol. 2018 Dec;58(6):686-9.

69 Ratzer MA. The influence of marriage, pregnancy and childbirth on acne vulgaris. Br J Dermatol. 1964 Apr;76(4):165-8.

70 Shaw JC, White LE. Persistent acne in adult women. Arch Dermatol. 2001 Sep;137(9): 1252-3.

71 Dréno B, Blouin E, Moyse D, Bodokh I, Knol AC, Khammari A. Acne in pregnant women: a French survey. Acta Derm Venereol. 2014 Jan;94(1):82-3.

72 Chien AL, Qi J, Rainer B, Sachs DL, Helfrich YR. Treatment of acne in pregnancy. J Am Board Fam Med. 2016 Mar-Apr;29(2):25462.

73 Murase JE, Heller MM, Butler DC. Safety of dermatologic medications in pregnancy and lactation: part I. Pregnancy. J Am Acad Dermatol. 2014 Mar;70(3):401.e1-14.

74 Trivedi MK, Kroumpouzos G, Murase JE. A review of the safety of cosmetic procedures during pregnancy and lactation. Int J Womens Dermatol. 2017 Feb;3(1):6-10.

75 Bechstein SK, Ochsendorf F. [Acne and rosacea in pregnancy]. Hautarzt. 2017 Feb;68(2) 111-9.

76 Jarrett R, Gonsalves R, Anstey AV. Differing obstetric outcomes of rosacea fulminans in pregnancy: report of three cases with review of pathogenesis and management. Clin Exp Dermatol. 2010 Dec;35(8):888-91.

77 Demir O, Tas IS, Gunay B, Ugurlucan FG. A rare dermatologic disease in pregnancy: rosacea fulminans - case report and review of the literature. Open Access Maced J Med Sci. 2018 Aug;6(8):1438-41.

78 Vossen AR, van Straalen KR, Prens EP, van der Zee HH. Menses and pregnancy affect symptoms in hidradenitis suppurativa: A cross-sectional study. J Am Acad Dermatol. 2017 Jan;76(1):155-6.

79 Perng P, Zampella JG, Okoye GA. Management of hidradenitis suppurativa in pregnancy. J Am Acad Dermatol. 2017 May;76(5): 979-89.

80 Yost J, Robinson M, Meehan SA. Fox-Fordyce disease. Dermatol Online J. 2012 Dec;18(12): 28.
81 Cornbleet T. Pregnancy and apocrine gland diseases: hidradenitis, Fox-Fordyce disease. AMA Arch Derm Syphilol. 1952 Jan;65(1):12-9.

82 Daneshpazhooh M, Chams-Davatchi C, Valikhani M, Aghabagheri A, Mortazavizadeh SM, Barzegari M, et al. Pemphigus and pregnancy: a 23-year experience. Indian J Dermatol Venereol Leprol. 2011 Jul-Aug;77(4):534

83 Lin L, Zeng X, Chen Q. Pemphigus and pregnancy. Analysis and summary of case reports over 49 years. Saudi Med J. 2015 Sep;36(9): 1033-8.

84 Çayırlı M, Tunca M, Akar A, Akpak YK. Favourable outcome of pregnancy in a patient with pemphigus vulgaris. J Obstet Gynaecol. 2015 Dec;35(7):747-8.

85 Elmuradi S, Ojeda D, Stoopler ET. Oral pemphigus vulgaris in pregnancy. J Obstet Gynaecol Can. 2015 Nov;37(11):951-2.

86 Rangel J. Pregnancy-associated "cutaneous type" pemphigus vulgaris. Perm J. 2016; 20(1):e101-2.

87 Vin H, Seyfer SJ, McClain CM, Hsu S. Concomitant pemphigus vulgaris and pemphigoid gestationis: a case report and review of the literature. Dermatol Online J. 2016 Jan; 22(1): 13030/qt2kk0h06d.

88 Kokolios M, Lamprou F, Stylianidou D, Sotiriadis D, Patsatsi A. New onset pemphigus foliaceus during pregnancy: A rare case. Int J Womens Dermatol. 2017 Dec;4(2):109-12.

89 Tavakolpour S, Mirsafaei HS, Delshad S. Management of pemphigus disease in pregnancy. Am J Reprod Immunol. 2017 Jan; 77(1):e12601.

90 Piccinni MP. T cells in pregnancy. Chem Immunol Allergy. 2005;89:3-9.

91 Zhu H, Chen Y, Zhou Y, Wang Y, Zheng J, Pan M. Cognate Th2-B cell interaction is essential for the autoantibody production in pemphigus vulgaris. J Clin Immunol. 2012 Feb;32(1):114-23.

92 Saito S. Cytokine network at the feto-maternal interface. J Reprod Immunol. 2000 Jul; 47(2):87-103.

93 Levi-Schaffer F, Klapholz L, Kupietzky A, Weinrauch L, Shalit M, Okon E. Increased numbers of mast cells in pemphigus vulgaris skin lesions. A histochemical study. Acta Derm Venereol. 1991 May;71(3):269-71.

94 Kalayciyan A, Engin B, Serdaroglu S, Mat C, Aydemir EH, Kotogyan A. A retrospective analysis of patients with pemphigus vulgaris associated with pregnancy. Br J Dermatol. 2002 Aug;147(2):396-7.

95 Gushi M, Yamamoto Y, Mine Y, Awazawa R, Nonaka K, Taira K, et al. Neonatal pemphigus vulgaris. J Dermatol. 2008 Aug;35(8):529-35.

96 Tope WD, Kamino H, Briggaman RA, Rico MJ, Prose NS. Neonatal pemphigus vulgaris in a child born to a woman in remission. J Am Acad Dermatol. 1993 Sep;29(3):480-5.

97 Bonifazi E, Milioto M, Trashlieva V, Ferrante MR, Mazzotta F, Coviello C. Neonatal pemphigus vulgaris passively transmitted from a clinically asymptomatic mother. J Am Acad Dermatol. 2006 Nov;55(5 Suppl):S113-4.
98 Wan J, Imadojemu S, Werth VP. Management of rheumatic and autoimmune blistering disease in pregnancy and postpartum. Clin Dermatol. 2016 May-Jun;34(3):344-52.

99 Kolstad KD, Fiorentino D, Li S, Chakravarty EF, Chung L. Pregnancy outcomes in adult patients with dermatomyositis and polymyositis. Semin Arthritis Rheum. 2018 Jun; 47(6):865-9.

100 Gutiérrez G, Dagnino R, Mintz G. Polymyositis/dermatomyositis and pregnancy. Arthritis Rheum. 1984 Mar;27(3):291-4.

101 King CR, Chow S. Dermatomyositis and pregnancy. Obstet Gynecol. 1985 Oct;66(4): 589-92.

102 Váncsa A, Ponyi A, Constantin T, Zeher M, Dankó K. Pregnancy outcome in idiopathic inflammatory myopathy. Rheumatol Int. 2007 Mar;27(5):435-9.

103 Taraborelli M, Ramoni V, Brucato A, Airò P, Bajocchi G, Bellisai F, et al.; IMPRESS Investigators. Brief report: successful pregnancies but a higher risk of preterm births in patients with systemic sclerosis: an Italian multicenter study. Arthritis Rheum. 2012 Jun; 64(6): 1970-7.

104 Lidar M, Langevitz P. Pregnancy issues in scleroderma. Autoimmun Rev. 2012 May; 11(6-7):A515-9.

105 Steen VD, Medsger TA Jr. Fertility and pregnancy outcome in women with systemic sclerosis. Arthritis Rheum. 1999 Apr;42(4): 763-8.

106 Chakravarty EF, Khanna D, Chung L. Pregnancy outcomes in systemic sclerosis, primary pulmonary hypertension, and sickle cell disease. Obstet Gynecol. 2008 Apr; 111(4):927-34.

107 Marder W, Littlejohn EA, Somers EC. Pregnancy and autoimmune connective tissue diseases. Best Pract Res Clin Rheumatol. 2016 Feb;30(1):63-80.

108 Lateef A, Petri M. Managing lupus patients during pregnancy. Best Pract Res Clin Rheumatol. 2013 Jun;27(3):435-47.

109 Barbhaiya M, Bermas BL. Evaluation and management of systemic lupus erythematosus and rheumatoid arthritis during pregnancy. Clin Immunol. 2013 Nov; 149(2): 225-35.

110 Borella E, Lojacono A, Gatto M, Andreoli L, Taglietti M, Iaccarino L, et al. Predictors of maternal and fetal complications in SLE patients: a prospective study. Immunol Res. 2014 Dec;60(2-3):170-6.

111 Eudy AM, Siega-Riz AM, Engel SM, Franceschini N, Howard AG, Clowse ME, et al. Effect of pregnancy on disease flares in patients with systemic lupus erythematosus. Ann Rheum Dis. 2018 Jun;77(6):855-60.

112 Khan A, Thomas M, P K SD. Pregnancy complicated by systemic lupus erythematosus and its outcome over 10 years. J Obstet Gynaecol. 2018 May;38(4):476-81.

113 Kannambal K, Tharini GK. A screening study on dermatoses in pregnancy. J Clin $\mathrm{Di}$ agn Res. 2017 May;11(5):WC01-05. 
114 Singh AG, Chowdhary VR. Pregnancy-related issues in women with systemic lupus erythematosus. Int J Rheum Dis. 2015 Feb; 18(2):172-81.

115 Tedeschi SK, Massarotti E, Guan H, Fine A, Bermas BL, Costenbader KH. Specific systemic lupus erythematosus disease manifestations in the six months prior to conception are associated with similar disease manifestations during pregnancy. Lupus. 2015 Oct; 24(12):1283-92.
116 Smyth A, Oliveira GH, Lahr BD, Bailey KR, Norby SM, Garovic VD. A systematic review and meta-analysis of pregnancy outcomes in patients with systemic lupus erythematosus and lupus nephritis. Clin J Am Soc Nephrol. 2010 Nov;5(11):2060-8.

117 Zhang C, Liang MY, Xu X, Zhang XW, Chen S. Clinical features of new-onset systemic lupus erythematosus in pregnant patients. J Obstet Gynaecol Res. 2018 Feb;44(2):23440.

118 Hamed HO, Ahmed SR, Alzolibani A, Kamal MM, Mostafa MS, Gamal RM, et al. Does cutaneous lupus erythematosus have more favorable pregnancy outcomes than systemic disease? A two-center study. Acta Obstet Gynecol Scand. 2013 Aug;92(8):93442.
119 Webb KC, Lyon S, Nardone B, West DP, Kundu RV. Influence of pregnancy on vitiligo activity. J Clin Aesthet Dermatol. 2016 Dec;9(12):21-5.

120 Horev A, Weintraub AY, Sergienko R, Wiznitzer A, Halevy S, Sheiner E. Pregnancy outcome in women with vitiligo. Int J Dermatol. 2011 Sep;50(9):1083-5.

121 Park KY, Kwon HJ, Wie JH, Lee HH, Cho SB, Kim BJ, et al. Pregnancy outcomes in patients with vitiligo: A nationwide population-based cohort study from Korea. J Am Acad Dermatol. 2018 Nov;79(5):836-42. 